\title{
UV- Irradiation Induced Synthesis of Fluorescent Poly (Acrylic Acid) Stabilized Silver Clusters
}

\author{
USMAN Ken Aldren S. ${ }^{1, a}$, TRINIDAD Lawrence John Paulo L. ${ }^{2, b}$, and \\ PAYAWAN Leon M. Jr. ${ }^{1,2, c}$ \\ ${ }^{1}$ Natural Sciences Research Institute, University of the Philippines, Diliman, Quezon City, Philippines \\ ${ }^{2}$ Institute of Chemistry, University of the Philippines, Diliman, Quezon City, Philippines
}

\begin{abstract}
Photochemical treatment using ultraviolet radiation was used to prepare fluorescent silver nanoparticles/nanoclusters from $\mathrm{AgNO}_{3}$ precursor upon its encapsulation with Poly (Acrylic Acid). Spectrofluorometric analysis showed an excitation spectra with maxima at approximately $450 \mathrm{~nm}$ and 550 regions nm when fixed wavelength of $600 \mathrm{~nm}$ was used to scan the solutions. Fluorescent emission occurred at around $500 \mathrm{~nm}$ and $700 \mathrm{~nm}$ using the $450 \mathrm{~nm}$ excitation wavelength. High molecular weight polymer (AgPAA1250) showed higher intensity of emission than low molecular weight (AgPAA450). Stability of the nanoparticle solution was assessed using Zeta Potential Measurements. Despite having a larger average particle diameter, Zeta Potential value for AgPAA 1250 is more negative than AgPAA 450, $-59.3 \mathrm{mV}$ and $-47.5 \mathrm{mV}$ respectively. This tells us that using a polymer with larger molecular weight can better prevent the aggregation of the nanoparticles.
\end{abstract}

\section{Introduction}

Several metal clusters have attracted scientists for their fluorescent-property [1]. Their fluorescence is mainly due to the inter-band electronic transition from the submerged and quasi-continuum $5 \mathrm{~d}$ band to the lowest unoccupied conduction band of the clusters [2]. Previous studies show that fluorescence was attributed to the coupling of photo-activated clusters and silver oxides [3]. Metal clusters like that of silver have absorption characteristic bands at $275 \mathrm{~nm}\left(\mathrm{Ag}_{4}\right), 300 \mathrm{~nm}$ (trimer), $330 \mathrm{~nm}$ (pentamer), and 345nm (heptamer) [4]. These are, however, short-lived and form aggregates after several hours [5]. These can then be stabilized by polymers via multi-coordination resulting to chemical adsorption of the polymer onto the metal surface. Polymers such as Poly (Methyl Methacrylate) and Poly (Acrylic Acid) have strong affinity for silver ions due to their carboxylic acid pendant group [6,7] and since these are FDA-approved water-soluble polymers they will have wide applications for bio-imaging. Radiolytic method is advantageous for the synthesis of this fluorescent material because the method is reproducible; minimum impurities are introduced, and reactions proceed homogeneously [8].

\section{Experimental}

\subsection{Synthesis of Ag/PAA nanocomposites}

Polymer-encapsulated silver was generated from UVirradiation of aqueous solutions of $\mathrm{AgNO}_{3}$ and Poly
(Acrylic Acid) (450 and $1250 \mathrm{KDa}$ ) as stabilizer. Dropwise additions of $4 \mathrm{~mL} 2.0 \times 10^{-3} \mathrm{M} \mathrm{AgNO}_{3}$ to $15 \mathrm{~mL}$ PAA solutions were done in an inert atmosphere. Another set of AgPAA solutions was prepared with additional 1 $\mathrm{mL}$ isopropanol (IPA). Solutions were then irradiated in a UV Consulting Peschl Photocatalytic Reactor for a span of 1 hour.

\subsection{Fluorescence spectroscopy}

Spectral scans of the nanocomposites were done using a Double-beam UV-vis Spectrophotometer (UV-1700 Series, Shimadzu) while the fluorescence were monitored using a Multi-mode Spectrofluorometer (Varioskan $^{\mathrm{TM}}$ Flash Multimode Reader, Thermoscientific).

\subsection{Particle distribution}

The particle distribution (Dynamic Light Scattering) and stability (Zeta Potential Measurements) of the nanocomposite solution were measured using Malvern Zeta Sizer Nano ZS90.

\section{Results and discussion}

Addition of the silver salt to the PAA solution caused the expanded coil of the polymer to collapse into globular state through the interaction of carboxylate group with silver cations $[9,10]$. This allows the negatively charged polymer to form a spherical shell around the positive

\footnotetext{
Corresponding author: ${ }^{\mathrm{a}}$ kenaldrenusman@yahoo.com; ${ }^{\mathrm{b}}$ liptrinidad@gmail.com; ${ }^{\mathrm{c}} \underline{\text { lmpayawan@yahoo.com }}$
} 
silver cations through electrostatic interaction [11] as shown in Figure 1.

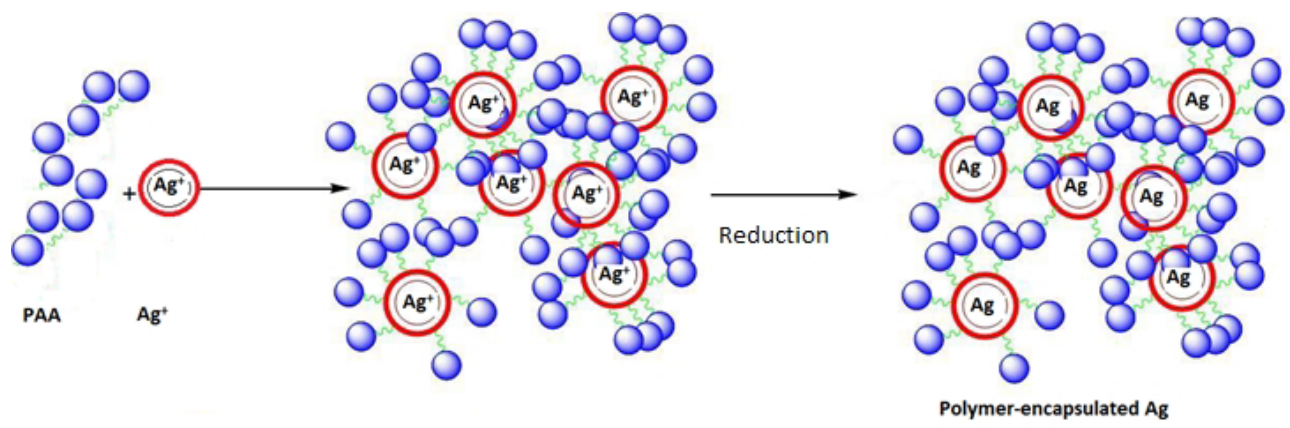

Figure 1. Schematic illustration of the process for the polymer protected metal nanoparticles.

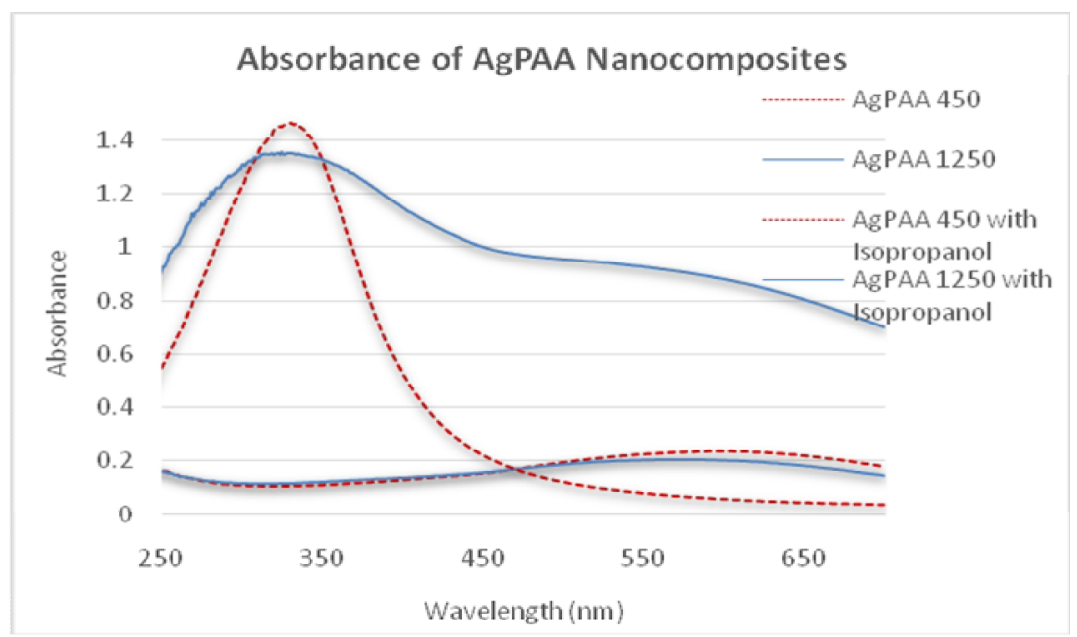

Figure 2. Absorption spectra of AgPAA Nanocomposites (with and without IPA).

\subsection{Absorption spectrum}

Clear solutions of AgPAA with additional isopropanol turned golden-brown in color while solutions without IPA changed to blue after UV-irradiation. Spectral scan of the solutions in Figure 2. showed absorption broad band at $400 \mathrm{~nm}$ and $600 \mathrm{~nm}$ for samples with and without IPA, respectively. The absorbance at $400 \mathrm{~nm}$ is the known silver nanoparticles surface plasmon band, which is also called the Mie resonance [6,7]. This then indicates that silver nanoparticles were not formed when IPA was not added. Instead, the absorbance at $600 \mathrm{~nm}$ corresponds to the formation of silver ion clusters. It is known that the high energy deposition from ultraviolet light throughout the solution leads to ionization, excitation and eventually to fragmentation of highly excited molecules forming free radical species such as solvated electrons $\left(\mathrm{e}_{\mathrm{aq}}^{-}\right)$, which reduce metal cations to metal atoms. However, reduction of $\mathrm{Ag}_{(\text {(aq) }}^{+}$to $\mathrm{Ag}_{(\mathrm{s})}$ was incomplete due to the formation of hydroxyl radicals from the cleavage of water molecules upon irradiation. IPA is known to act as a radical scavenger since it produces secondary carbon and hydrogen radicals wherein the latter reacts with the hydroxyl radicals and produces water as a byproduct. Thus in the absence of isopropanol, the hydroxyl radicals, which are known to be strong oxidizing agents, compete with the reduction of the $\mathrm{Ag}^{+}$, then this process was subsequently followed by slow aggregation of isolated atoms $\left(\mathrm{Ag}_{\mathrm{n}}\right)$ [8]. Coalescence of neighboring watersoluble clusters is inhibited at early stages by Poly(Acrylic Acid) (PAA) as it forms spherical structures surrounding the metal nanoparticles $[9,10]$. 

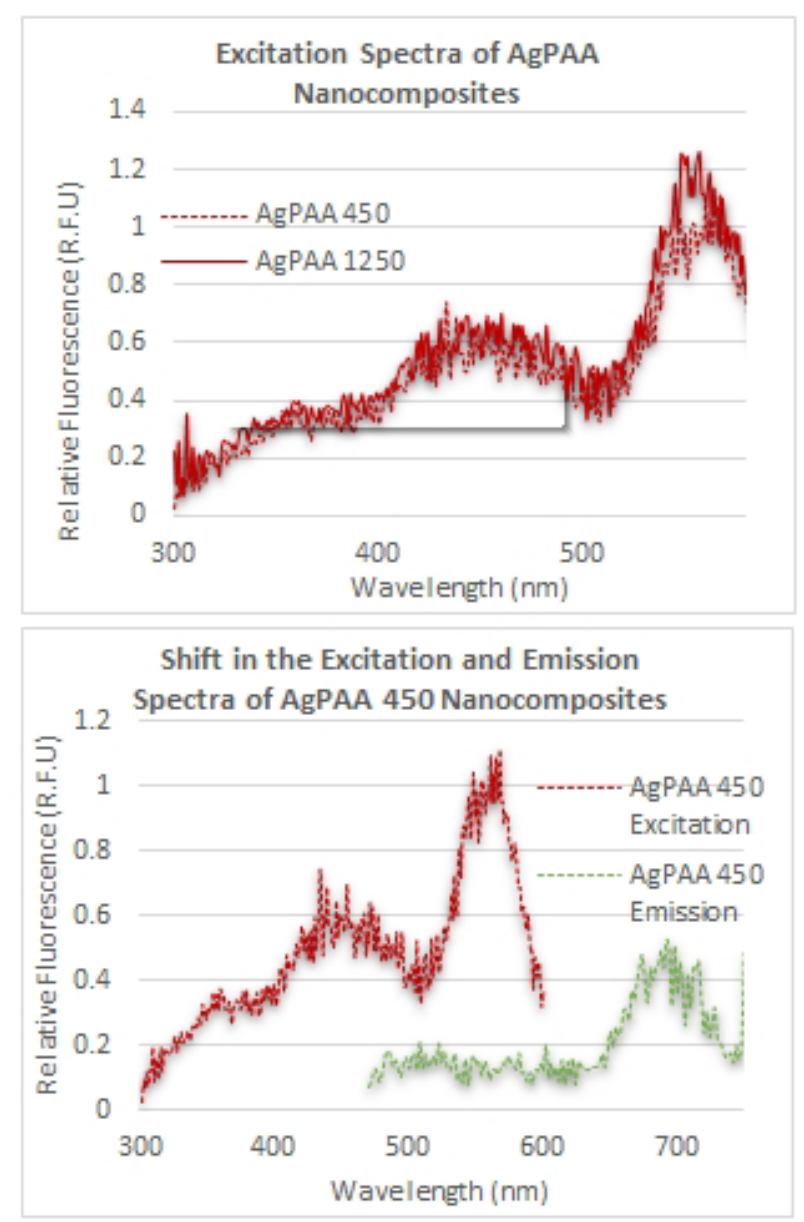
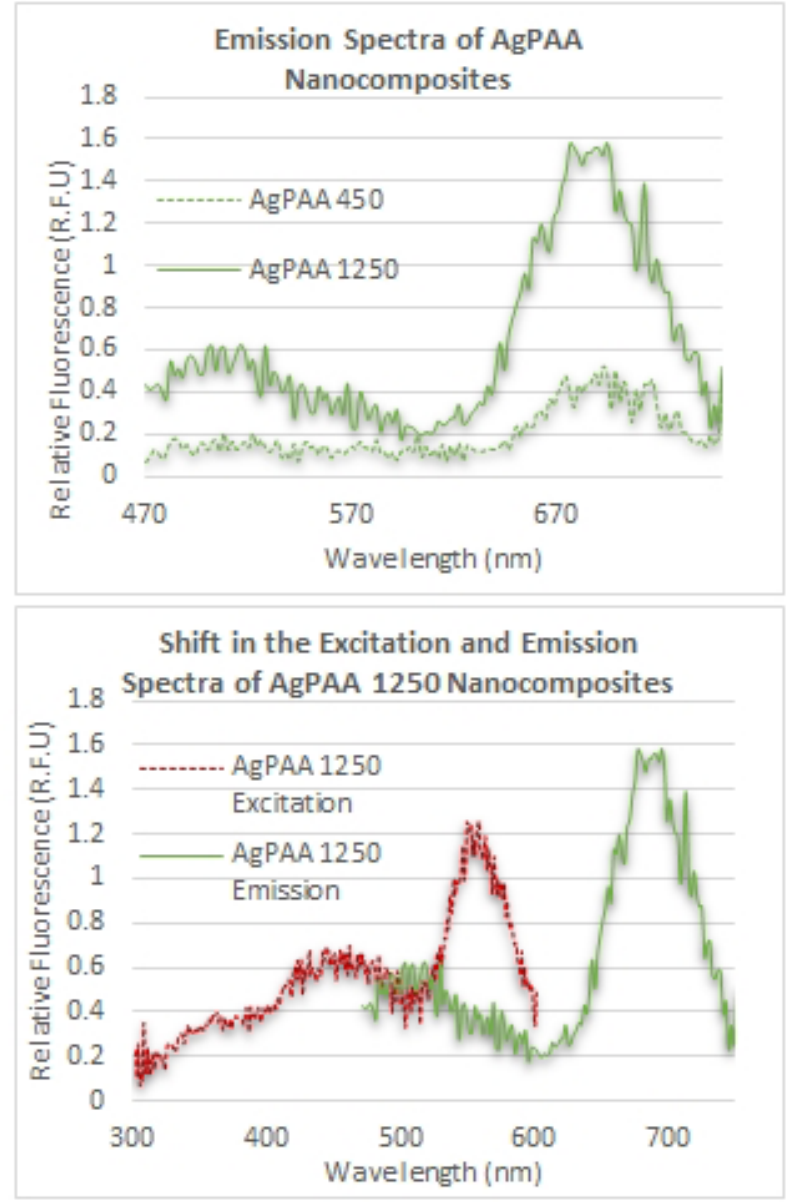

Figure 3. (Top Left) Excitation spectra obtained from the fixed wavelength scans at $600 \mathrm{~nm}$. Excitation wavelengths obtained are 450 $\mathrm{nm}$ and $550 \mathrm{~nm}$ respectively. (Top Right) Comparison of the Emission spectra obtained from the fixed wavelength scans of irradiated Ag/PAA solution of MW $450 \mathrm{kDa}$ (shown as lines) and $1250 \mathrm{kDa}$ (round dots) using $450 \mathrm{~nm}$ Excitation wavelength. (Bottom) Stokes' Shift in the Excitation and Emission Spectra of AgPAA 450 (left) and AgPAA 1250 (Right). **AgPAA solutions with additional IPA did not exhibit Fluorescence.

\subsection{Analysis of the fluorescence spectra (excitation and emission)}

Excitation spectra of irradiated solution were obtained using $\lambda=600 \mathrm{~nm}$, the wavelength of maximum absorbance for the solutions. However, two peaks were obtained from the excitation spectrum for both wavelengths, namely, peaks at $450 \mathrm{~nm}$ and $550 \mathrm{~nm}$. The maxima at $450 \mathrm{~nm}$ was then used as the excitation wavelength in order to obtain the emission spectra of the solutions, which is similar to the absorption of charged oligomeric silver cluster (Treguer, et al., 2005). Two fluorescence bands were then observed: (1) a small band around $500 \mathrm{~nm}$ and (2) a large fluorescence band around $700 \mathrm{~nm}$. Stokes' Shift was observed upon comparison between the obtained excitation and emission spectra. This tells us about the re-emission of longer wavelength photons (500 $\mathrm{nm}$ and $700 \mathrm{~nm}$ ) by a molecule/particle that has absorbed photons of shorter wavelengths $(450 \mathrm{~nm}$ and $550 \mathrm{~nm}$ ). However, the obtained emission spectrum is not a mirror image of the excitation spectra as shown in Figure 3. This could be attributed to factors such as absorption of the fluorescence by other species in the solution, molecular aggregation, dimerization of silver clusters/formation of various silver clusters and scattering of light. Another observed variance is the weaker fluorescence of silver stabilized with $450 \mathrm{kDa}$ polymer compare to $1,250 \mathrm{kDa}$ polymer. This tells us that polymers with larger MW can stabilize the silver cluster more efficiently as compared to polymers with smaller MW. This assumption was then confirmed by measuring the particle size and zeta potential of the particles in the solution using Dynamic Light Scattering and Zeta Potential Measurements respectively.

\subsection{Dynamic light scattering and zeta potential measurements}

Poly (Acrylic Acid) with a larger polymer molecular weight also showed better encapsulation of the nanoparticles. This was supported by the Dynamic Light Scattering (DLS) and Zeta Potential Measurement results shown in Table 1. 
Table 1. Summary of dynamic light scattering and Zeta potential measurement results.

\begin{tabular}{ccccc}
\hline & $\begin{array}{c}\text { Size } \\
(\mathbf{n m})\end{array}$ & $\begin{array}{c}\text { Zeta Potential } \\
(\mathbf{m V})\end{array}$ & $\begin{array}{c}\text { Conductivity } \\
(\mathbf{m S} / \mathbf{c m})\end{array}$ & $\begin{array}{c}\text { Electrophoretic } \\
\text { Mobility }(\boldsymbol{\mu m c m} / \mathbf{V s})\end{array}$ \\
\hline AgPAA 450 & 208.4 & -47.5 & 0.216 & -3.724 \\
\hline AgPAA 1250 & 243.6 & -59.3 & 0.307 & -4.65 \\
\hline
\end{tabular}

Average particle diameter for the two samples were $208.4 \mathrm{~nm}$ for $\mathrm{Ag} / \mathrm{PAA} 450 \mathrm{kDa}$ and $243.6 \mathrm{~nm}$ for $\mathrm{Ag} / \mathrm{PAA} 1250 \mathrm{kDa}$. This is because high MW polymer can form necklace structures which serve as attachments for other polymer chains, resulting in train-like structures [11] and thus, larger particle size. Nonetheless, the solution stabilized by larger MW polymer had better stability in terms of Zeta Potential Values, as shown in Table 1. AgPAA 1250 also had higher Conductivity and Electrophoretic Mobility. These attributes are due to the presence of more carboxylate functional groups for the larger molecular weight polymer. The carboxylate group in theory then causes the repulsion of the encapsulated silver particles towards other silver species in the solution, which prevents the cluster aggregation [11]. Presence of more negative carboxylate groups around the silver clusters resulted to a more negative value of zeta potential and better conductivity and electrophoretic mobility.

\section{Conclusions}

Solution of UV-irradiated Poly(Acrylic Acid)-stabilized silver nanoclusters gave high intensity fluorescence around the $500 \mathrm{~nm}$ and $700 \mathrm{~nm}$ region which resulted in the formation of water-soluble silver clusters. $\mathrm{Ag}$ particles stabilized with polymer larger MW exhibited stronger emission as compared to those stabilized with smaller polymer. Additionally, the emission spectra did not appear to be a mirror image of the excitation spectra, which is a characteristic of an oligomeric silver cluster solution. The difference in the intensity of the emission and excitation could be attributed to aggregation and light absorption of other non-fluorescent clusters/species in the solution. Characterization techniques to specify the components of the cluster could then be explored for future studies in order to specify which species exhibited fluorescence. It could also be interesting to see if we could incorporate biochemical samples into these clusters and explore its interaction for a certain target molecule (fluorescent tagging).

\section{Acknowledgements}

The authors wish to thank Natural Sciences Research Institute for funding the project (Code: CHE-13-2-04) and the Institute of Chemistry for the use of the needed equipment.

\section{References}

1. H. Xu, K.S. Suslick, Advanced Materials 22, 10781082 (2010)

2. Y. Dai, X. Hu, C. Wang, D. Chen, X. Jiang, C. Zhu, B. Yu, J. Qiu, Chem. Phys.Lett. 439, 81-84 (2007)

3. A. Maali, T. Cardinal, M. Treguer-Delapierre, Physics E. 17, 559-560 (2003)

4. A. Henglein, Chem. Phys. Lett. 154, 473 (1989)

5. B.G. Ershov, A. Henglein, J. Phys. Chem. B 102, 10663-10667 (1998)

6. L. Shang, S. Dong, Commun. Camb. 9, 1088-1090 (2008)

7. N. Toshima, Y. Shiraishi, T. Teranishi, Journal of Molecular Catalysis A: Chemical 177, 139-147 (2001)

8. Treguer, et al., Solid State Sciences 7, 812-818 (2005)

9. K.A.S. Usman, L.J.P. Trinidad, L.M. Payawan Jr., Advanced Materials Research 1098, 19-24 (2015)

10. K.A. Usman, A. Rosales, L. Payawan Jr., Journal of the Kapisanang Kimika ng Pilipinas (Chemical Society of the Philippines, Inc.) 25, 2-11 (2014)

11. A. dela Santa, National Library of Canada, 26189629 (2002) 